\title{
MicroRNA-29a promotes the neural differentiation of rat neural stem/progenitor cells by targeting KLF4
}

\author{
YUNAN GAO ${ }^{1,2}$, HU QIAO ${ }^{1,2}$, TIANYU ZHONG ${ }^{1,2}$, ZHEN LU $^{3}$ and YUXIA HOU ${ }^{1,2}$ \\ ${ }^{1}$ Key Laboratory of Shaanxi Province for Craniofacial Precision Medicine Research, College of Stomatology, \\ Xi'an Jiaotong University; ${ }^{2}$ Department of Orthodontics, Stomatological Hospital of Xi'an Jiaotong University, Xi'an, \\ Shaanxi 710004; ${ }^{3}$ Department of Orthodontics, The First Affiliated Hospital of \\ Xi'an Jiaotong University, Xi'an, Shaanxi 710061, P.R. China
}

Received November 2, 2019; Accepted April 23, 2020

DOI: $10.3892 / \mathrm{mmr} .2020 .11177$

\begin{abstract}
Neural stem/progenitor cells (NSPCs) remain in the mammalian brain throughout life, where they have the ability to self-renew and generate different types of cell in the central nervous system (CNS). Therefore, NSPCs may be a potential novel therapeutic strategy following damage to the CNS. Previous research has reported that microRNA (miR)-29a served an important role in regulating cell proliferation, differentiation and survival; however, to the best of our knowledge, little is known of the effect of miR-29a in neural differentiation. The present study aimed to investigate the effect of miR-29a on the differentiation of NSPCs, determined via RNA interference, immunostaining, reverse transcription-quantitative PCR and western blotting. The present study discovered that the expression levels of miR-29a were significantly upregulated in a time-dependent manner during neural differentiation. Immunostaining showed that overexpression of miR-29a promoted neural differentiation, which manifested in increased expression levels of neuron-specific class III $\beta$-tubulin (Tuj1); however, miR-29a had no effect on neuroglial differentiation. The expression levels of Kruppel-like factor 4 (KLF4) were downregulated following overexpression of miR-29a, whereas the inhibition of miR-29a demonstrated the opposite effect. These results suggested that the overexpression of miR-29a may promote neural differentiation in cultured rat NSPCs by decreasing the expression levels of KLF4. Thus indicating that
\end{abstract}

Correspondence to: Professor Yuxia Hou, Key Laboratory of Shaanxi Province for Craniofacial Precision Medicine Research, College of Stomatology, Xi'an Jiaotong University, 98 Xiwu Road, Xi'an, Shaanxi 710004, P.R. China

E-mail: 369hyx@163.com

Abbreviations: AD, Alzheimer's disease; bFGF, basic fibroblast growth factor; CNS, central nervous system; EGF, epidermal growth factor; KLF4, Kruppel-like factor 4; NSPCs, neural stem/progenitor cells; PD, Parkinson's disease; PDL, poly-D-lysine

Key words: neural stem/progenitor cells, microRNA-29a, neural differentiation, Kruppel-like factor 4 targeting KLF4, a crucial regulatory factor for the maintenance of stemness, may be a potential underlying mechanism of action for miR-29a. In conclusion, the findings of the present study identified a potential mechanism of action for miR-29a in NSPC differentiation and provided a novel insight into the treatment strategies for CNS damage.

\section{Introduction}

Neural stem/progenitor cells (NSPCs) remain in the mammalian central nervous system (CNS) throughout life, where they have the ability to self-renew and differentiate into multiple different types of cell (1). In the postnatal brain, the vast majority of NSPCs are spatially restricted to two specific brain regions: The subventricular zone of the lateral ventricles and the subgranular zone in the dentate gyrus of the hippocampus (2). Previous studies have indicated that postnatal NSPCs may be activated in response to pathophysiological stimuli, such as cerebral hemorrhage, traumatic brain injury and stroke, and that they may participate in CNS damage repair and functional recovery (3-5). Therefore, NSPC replacement therapy may aid in the development of new treatment modalities for diseases of the CNS. Nonetheless, this technique is restricted by the number of NSPCs and newly generated neurons in the brain, thus it is currently difficult to use NSPCs for clinical therapy (6). Therefore, there remains an urgent requirement to identify the regulatory functions of NSPCs that permit their positive enrichment in the CNS.

Numerous factors, including intracellular signal molecules, the extracellular niche and microRNAs (miRNAs/miRs) have been discovered to be involved in regulating the proliferation, differentiation and maintenance of NSPCs (7-9). For example, as a novel regulatory factor, miR-29a demonstrated huge potential over the control of cell behavior not only in cancer cells, but also in stem/progenitor cells $(10,11)$. More importantly, our previous study revealed that the overexpression of miR-29 promoted the proliferation of cultured rat NSPCs (12). These findings indicated that miR-29a may be an important indicator of regulatory factors in NSPC development. However, to the best of our knowledge, very little is known about the function of miR-29a in NSPC differentiation, let alone its intracellular signaling mechanisms. 
The present study aimed to investigate the effect of miR-29a on the differentiation of NSPCs. The finding of the study suggested that the overexpression of miR-29a may promote neural differentiation, whilst having little influence on astrocyte differentiation, potentially through regulating the zinc-finger transcription factor Kruppel-like factor 4 (KLF4). This research may offer novel insights into the onset of neurodevelopment and provide a potential therapeutic target for the treatment of diseases of the CNS.

\section{Materials and methods}

Rat NSPC culture. Rat NSPCs were prepared from E14.5 Sprague-Dawley rat embryos, which were obtained from pregnant Sprague-Dawley rats (certificate no. 22-9601018; Experimental Animal Center of Xi'an Jiantong University Health Science Center) as previously described with minor modifications $(13,14)$. A total of 5 pregnant Sprague-Dawley rats (age, 12 weeks; weight, 386-422 g) were used. All rats were maintained at $23 \pm 2^{\circ} \mathrm{C}$ on a 12-h light/dark cycle with free access to standard rat food and water. NSPCs were incubated in a humidified atmosphere of $5 \% \mathrm{CO}_{2}$ and $95 \%$ air at $37^{\circ} \mathrm{C}$, and cultured in serum-free complete medium, consisting of DMEM/F12 (1:1; Thermo Fisher Scientific, Inc.), $1 \% \mathrm{~N}-2$ supplement (cat. no. 17502048; Thermo Fisher Scientific, Inc.), 2\% B-27 supplement (cat. no. 17504044; Thermo Fisher Scientific, Inc.), $20 \mathrm{ng} / \mathrm{ml}$ epidermal growth factor (EGF; Thermo Fisher Scientific, Inc.) and $10 \mathrm{ng} / \mathrm{ml}$ basic fibroblast growth factor (bFGF; Thermo Fisher Scientific, Inc.) The differentiation medium was the same as the complete medium but did not contain bFGF and EGF. After 3-5 days of culture, suspended neurospheres of $80-200-\mu \mathrm{m}$ in diameter were observed in the medium using a binocular compound microscope (cat. no. CX200; Olympus Corporation) (magnification, x10). The neurospheres were subsequently dissociated using TrypLE (Invitrogen; Thermo Fisher Scientific, Inc.) into single NSPCs, which were used for follow-up studies. For single-cell adhesive culture, single NSPCs in the differentiation medium were allowed to attach onto poly-D-lysine (PDL)-coated coverslips at $37^{\circ} \mathrm{C}$. All experimental protocols were approved by the Animal Care and Use Regulation of Xi'an Jiaotong University Health Science Center. All efforts were made to minimize the suffering of the animals and to keep the numbers of animals used to a minimum.

Cell transfection. NSPCs were plated in PDL-coated 24-well or 6-well plates and transfected with $50 \mathrm{nM}$ miRNA or small interfering RNA (siRNA) for $6 \mathrm{~h}$ at $37^{\circ} \mathrm{C}$, then cells were cultured in normal differentiation medium for 3 days prior to use in subsequent experiments. The cells plated into the 24-well plates were used for immunofluorescence staining, whereas the cells plated into the 6-well plates were used for the reverse transcription-quantitative (RT-q)PCR and western blotting experiments. Transient transfections were performed using Lipofectamine ${ }^{\circledR} 2000$ reagent (Invitrogen; Thermo Fisher Scientific, Inc.), according to the manufacturer's protocol, and equivalent transfection medium was used as a control.

The miR-29a mimic-negative control (NC), miR-29a mimic, anti-miR-29a-NC, anti-miR-29a, miR-200c, miR-200c-NC,
siRNA targeting KLF4 (siKLF4) and non-specific siRNA (siNC) were purchased from Shanghai GenePharma Co., Ltd. All siRNA was pre-labeled by the supplier with fluorescent dye (fluorescein amidite, FAM). The target sequences were as follows: miR-29a mimic, 5'-ACUGAUUUCUUUUGG UGUUCAG-3'; miR-29a mimic-NC, 5'-UUCUCCGAACGU GUCACGUTT-3'; anti-miR-29a, 5'-ACTGATTTCAAAT GGTGCT-3'; anti-miR-29a-NC, 5'-UUGUACUACACAAAA GUACUG-3'; miR-200c, 5'-CGUCUUACCCAGCAGUGU UUG-3'; miR-200c-NC, 5'-UCACAACCUCCUAGAAAG AGUAGA-3'; siKLF4, 5'-UCCAAAGAAGAAGGAUCU CUU-3'; and siNC, 5'-CGTACGCGGAATACTTCGATT-3'. Transfection efficiency was examined using a fluorescence inverted microscope (magnification, x10) (DMI3000B; Leica Microsystems, Inc). Knockdown of KLF4 expression levels was further evaluated via western blotting.

Dual-luciferase reporter assay. The dual-luciferase reporter assay was performed as previously described (15). Briefly, the sequence of the KLF4 3' untranslated region (UTR) was cloned into the pSICHECK2 vector (Promega Corporation), and the following KLF4 primer: Forward, 5'-GCCTCGAGA TCCCAGACAGTGGATAT-3' and reverse, 5'-GCGCGGCCG CTTCAGATAAAATATTAT-3'. Cells were co-transfected with plasmid for $6 \mathrm{~h}$ at $37^{\circ} \mathrm{C}$ in the presence of Lipofectamine 2000, according to the manufacturer's instructions (Invitrogen; Thermo Fisher Scientific, Inc.). After $48 \mathrm{~h}$ incubation, the media was removed and the cells were lysed in a $1.5-\mathrm{ml}$ Eppendorf tube using a Dual-Luciferase Reporter assay system (Promega Corporation), according to the manufacturer's protocol. Fluorescence was detected using a microtiter plate reader (BioTek Instruments, Inc). Relative luciferase activity was determined by normalizing the Firefly luciferase activity (Flu value) to the Renilla luciferase activity (Rlu value). Each experiment was performed in triplicate. The Flu/Rlu value was calculated to obtain a mean of three independent experiments.

$R T-q P C R$. RT-qPCR was performed as previously described with minor modifications (12). Total RNA was isolated from NSPCs using TRIzol ${ }^{\circledR}$ reagent (Invitrogen; Thermo Fisher Scientific, Inc.), according to the manufacturer's protocol. Total RNA (1 $\mu \mathrm{g})$ was reverse transcribed into cDNA using Megaplexä Primer Pools (Applied Biosystems; Thermo Fisher Scientific, Inc.) and the TaqManä MicroRNA Reverse Transcription kit (Applied Biosystems; Thermo Fisher Scientific, Inc.). All RT reactions were carried out in triplicate in a Mastercycler ep gradient $\mathrm{S}$ cycler (Eppendorf) at $16^{\circ} \mathrm{C}$ for $30 \mathrm{~min}, 42^{\circ} \mathrm{C}$ for $30 \mathrm{~min}$ and $85^{\circ} \mathrm{C}$ for $5 \mathrm{~min}$. qPCR was subsequently performed using single tube $\operatorname{TaqMan}^{\circledR}$ MicroRNA assays and TaqMan ${ }^{\circledR} 2 \mathrm{X}$ Universal PCR Master mix, No AmpErase ${ }^{\circledR}$ UNG (cat. no. 4324018; Applied Biosystems; Thermo Fisher Scientific, Inc.), according to the manufacturers' protocols, in $10 \mu \mathrm{l}$ reactions. All the reactions were performed in triplicate in an iQ5 Real-Time PCR Detection system (Bio-Rad Laboratories, Inc.). To analyze the expression levels of KLF4 and GAPDH, total RNA $(1 \mu \mathrm{g})$ was reverse transcribed into cDNA using a RevertAid First Strand cDNA synthesis kit (Fermentas; Thermo Fisher Scientific, Inc.) supplemented with a mix of OligodT and random primers. qPCR was subsequently performed using a GoTaq qPCR 
Master mix (Roche Diagnostics $\mathrm{GmbH}$ ). The primer pairs used for the qPCR, synthesized by Takara Biotechnology Co., Ltd., were as follows: miR-29a forward, 5'-AGTGAAT GAGGCCTTCGAGA-3' and reverse, 5'-GCATCTGAGTCG CCACTGTA-3'; miR-200c forward, 5'-GGTTGCCCACTG GAAGAACACAAT-3' and reverse, 5'-TAGACAATCCCA AGGCCAAGGTCTG-3'; KLF4 forward, 5'-GAAATTCGC CCGCTCCGATGA-3' and reverse, 5'-CTGTGTGTTTGC GGTAGTGCC-3'; U6 forward, 5'-GCATCTGAGTCGCCA CTGTA-3' and reverse, 5'-CGCTTCACGAATTTGCGTGTC AT-3'; and GAPDH forward, 5'-CATCACTGCCACCCAGAA GACTG-3' and reverse, 5'-ATGCCAGTGAGCTTCCCGTTC AG-3'. The following thermocycling conditions were used for the qPCR: Initial denaturation at $95^{\circ} \mathrm{C}$ for $5 \mathrm{~min}$; followed by 40 cycles at $95^{\circ} \mathrm{C}$ for $10 \mathrm{sec}$ and $60^{\circ} \mathrm{C}$ for $1 \mathrm{~min}$. Expression levels were analyzed using the $2^{-\Delta \Delta \mathrm{Cq}}$ method (16).

Immunofluorescence. The immunofluorescence procedure was conducted according to a previous study (17). Briefly, following transfection and incubating for 3 days, NSPCs plated onto PDL-coated coverslips were fixed using $4 \%$ paraformaldehyde for $30 \mathrm{~min}$ at room temperature and washed twice with PBS. The cells were subsequently permeabilized with $0.3 \%$ Triton X-100 (diluted with PBS) and blocked with $10 \%$ normal donkey serum (Sigma-Aldrich; Merck KGaA) for $1 \mathrm{~h}$ at room temperature. The cells were incubated with the following primary antibodies diluted in PBS which contained $5 \%$ normal donkey serum overnight at $4^{\circ} \mathrm{C}$ : Anti-nestin (1:200; cat. no. MAB353; EMD Millipore), anti-neuron-specific class III $\beta$-tubulin (Tuj1; 1:200; cat. no. ab78078; Abcam), anti-glial fibrillary acidic protein (GFAP; 1:200; cat. no. ab7260; Abcam). Following the primary antibody incubation, the cells were washed with PBS and incubated with anti-mouse IgG ReadyProbesä secondary antibody, Alexa Fluor 488 (1:500; cat. no. R37114; Invitrogen; Thermo Fisher Scientific, Inc.) or anti-rabbit IgG ReadyProbes ${ }^{\mathrm{TM}}$ secondary antibody, Alexa Fluor 594 (1:500; cat. no. R37119; Invitrogen; Thermo Fisher Scientific, Inc.) for $2 \mathrm{~h}$ at room temperature. The nuclei were counterstained with $1 \mu \mathrm{g} / \mathrm{ml}$ DAPI at room temperature for $10 \mathrm{~min}$. Fluorescence images were observed and counted using a BX51 fluorescence microscope equipped with a DP70 digital camera (magnification, x400; Olympus Corporation) in 5 randomly selected fields of view. For semi-quantification, Image-Pro Plus 5.1 software (Media Cybernectics, Inc.) was used. The percentage of labeled cells was calculated and normalized by DAPI nuclei staining. A total of three independently prepared NSPC cultures were used for each assay. The experiments were performed in triplicate and repeated independently $\geq 3$ times.

Western blotting. At each end of the experiment, following transfection and incubating for 3 days, total protein was extracted by using RIPA lysis buffer (Pierce; Thermo Fisher Scientific, Inc.) complemented with a Protease Inhibitor Cocktail (Roche Diagnostics $\mathrm{GmbH}$ ). Total protein was quantified using a Bradford assay bicinchoninic acid assay (Pierce; Thermo Fisher Scientific, Inc.) and 20-40 $\mu \mathrm{g}$ protein/lane (20 $\mu \mathrm{g}$ protein was used for GFAP and $\beta$-Actin, $40 \mu \mathrm{g}$ protein was used for remaining test) was subjected to $12 \%$ SDS-PAGE. The separated proteins were subsequently transferred onto
PVDF membranes and blocked with 5\% non-fat dry milk at room temperature for $2 \mathrm{~h}$. The membranes were incubated with the following primary antibodies: Anti-Tuj1 (1:800), anti-KLF4 $(1: 1,000)$, anti-GFAP $(1: 1,000)$ and anti- $\beta$-actin (1:5,000; cat. no. A1978; Sigma-Aldrich; Merck KGaA) overnight at $4^{\circ} \mathrm{C}$. Following the primary antibody incubation, the membranes were incubated at room temperature for $2 \mathrm{~h}$. with a horseradish peroxidase-conjugated anti-rabbit IgG secondary antibody (1:10,000; cat. no. AP307P; Sigma-Aldrich; Merck $\mathrm{KGaA}$ ). Protein bands were visualized using an enhanced chemiluminescent substrate (Pierce; Thermo Fisher Scientific, Inc.), exposure to a Fuji X-ray film (Fujian Gutian Yuanhang Medical Co., Ltd.) and a G:Box gel imaging system (Syngene Europe, CHEMI-XT16). The expression levels were semi-quantified using ImageJ 3.5 software (National Institutes of Health).

Statistical analysis. All statistical analyses were performed using SPSS version 12.0 software (SPSS, Inc.) and the data are presented as the mean $\pm \mathrm{SD}$ from $\geq 3$ independent in vitro experiments. Statistical differences between groups were analyzed using a one-way ANOVA, followed by Tukey's post hoc test for multiple comparisons. $\mathrm{P}<0.05$ was considered to indicate a statistically significant difference.

\section{Results}

miR-29a is overexpressed during NSPC differentiation. Following the culture of primary rat NSPCs for 3 days, $\sim 100-\mu \mathrm{m}$ diameter neurospheres were observed in the medium (Fig. 1A). Subsequently, the neurospheres were dissociated into single cells and plated onto PDL-coated coverslips for immunofluorescence analysis (Fig. 1B); $\geq 95 \%$ of cells were discovered to express nestin, a specific marker of NSPCs (data not shown) (18). To observe the differentiation of NSPCs, single cells were plated onto PDL-coated coverslips and cultured in normal differentiation medium. Both Tuj1-(a marker of immature neurons) and GFAP-(a marker of astrocytes) positive cells were observed following immunofluorescence staining (Fig. 1C). To determine the expression levels of miR-29a during NSPC differentiation, the expression levels of miR-29a were analyzed using RT-qPCR analysis. The results revealed that the expression levels of miR-29a increased in a time-dependent manner (Fig. 1D), indicating that the expression levels of miR-29a may increase during NSPC differentiation. There were little differences observed between the miR-29a expression levels in NSPCs at days 3, 5 and 7, thus cells cultured in normal differentiation medium for 3 days was used in subsequent experiments.

Effects of miR-29a on NSPC differentiation. To investigate the effects of miR-29a on NSPC differentiation in cultured rat NSPCs, cells were transfected with miR-NC, miR-29a mimic, anti-miR-29a or anti-miR-29a-NC. Following transfection, the cells were cultured in normal differentiation medium for 3 days and the expression levels of miR-29a were detected using RT-qPCR analysis. The results indicated that compared with the control (Ctrl) group, both the NCs exerted no effect on the expression levels of miR-29a (Fig. 2A). Notably, following the transfection with the miR-29a mimic, the expression levels 

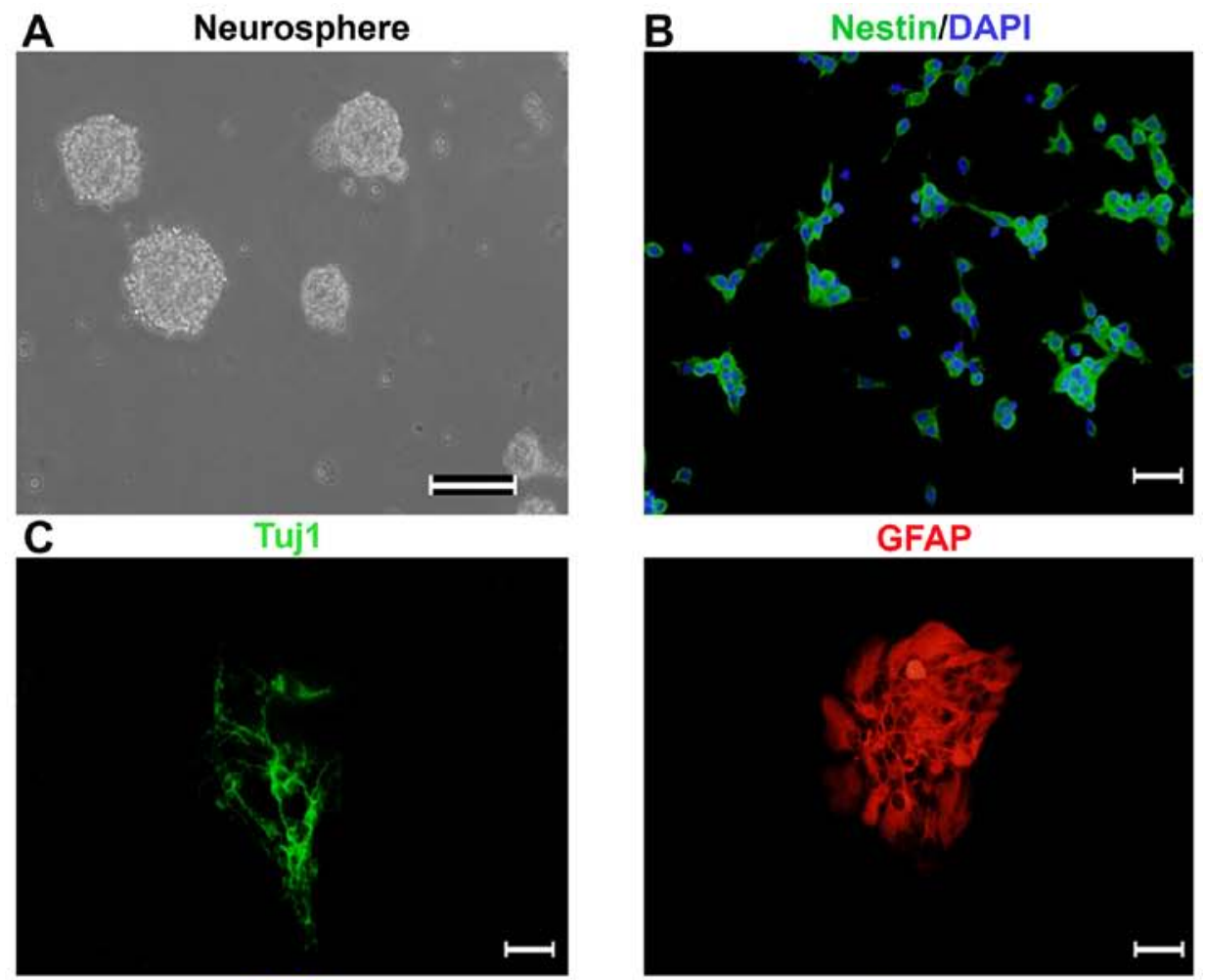

DAPI

Merged
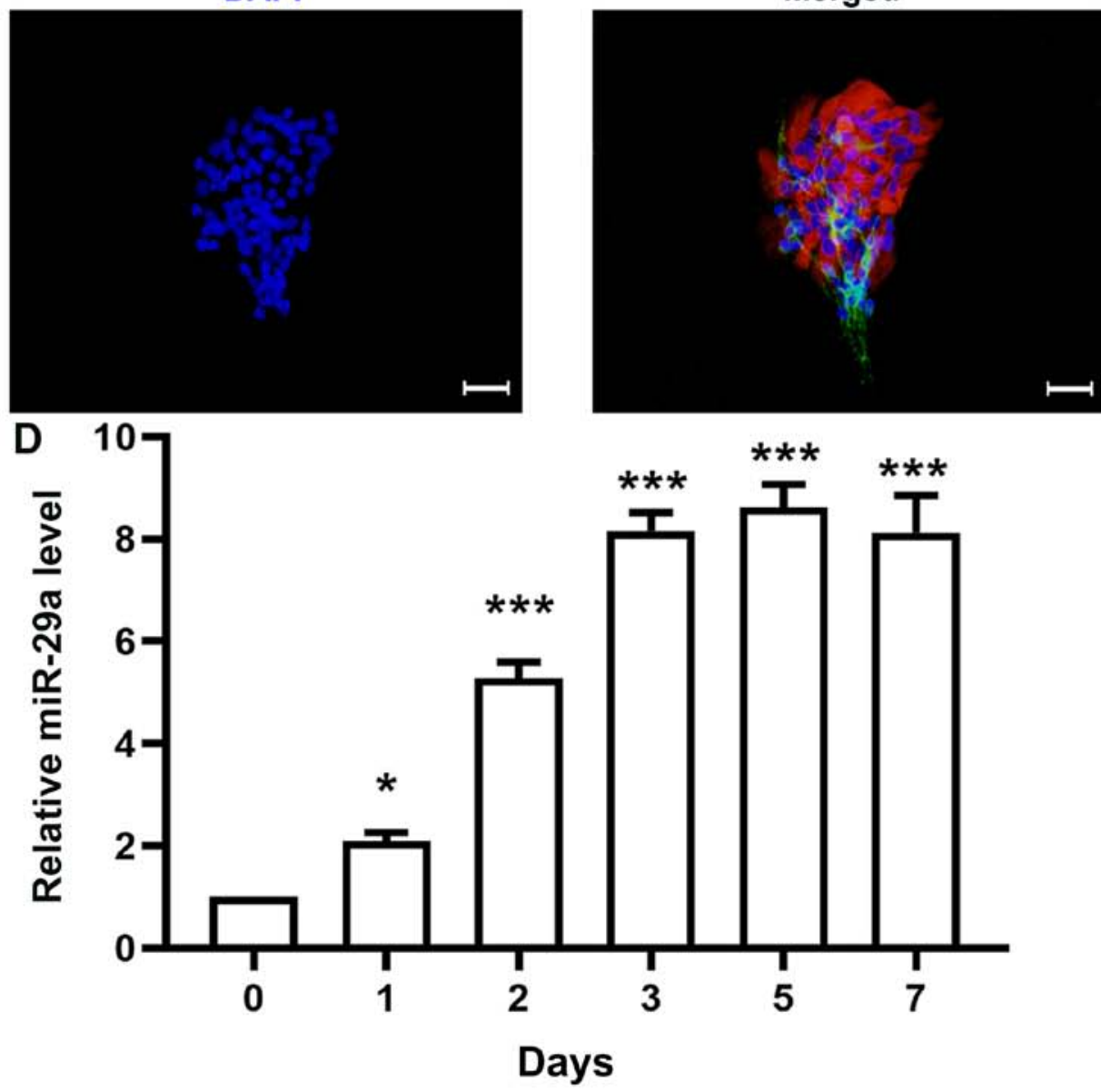

Figure 1. Expression levels of miR-29a are upregulated during neural differentiation. (A) NSPCs were dissected from E14.5 fetal rat cortexes. Neurospheres of $\sim 100-\mu \mathrm{m}$ in diameter were observed following culture for 3 days. Scale bar, $100 \mu \mathrm{m}$. (B) Single NSPCs were dissociated using TrypLE and cultured on the poly-D-lysine-coated slides. NSPCs were identified by nestin (green) immunofluorescence staining. Scale bar, $50 \mu \mathrm{m}$. (C) Both Tuj1-(green) and GFAP-(red) positive cells were visualized using immunostaining. Scale bar, $50 \mu \mathrm{m}$. (D) Reverse transcription-quantitative PCR analysis revealed that the expression levels of miR-29a during neuronal differentiation in NSPCs were upregulated in a time-dependent manner. Data are presented as the mean \pm SD of three independent experiments. ${ }^{*} \mathrm{P}<0.05,{ }^{* * *} \mathrm{P}<0.001$ vs. control ( $\left.0 \mathrm{~d}\right)$. Tuj1, neuron-specific class III $\beta$-tubulin; GFAP, glial fibrillary acidic protein; miR, microRNA; NSPCs, neural stem/progenitor cells. 


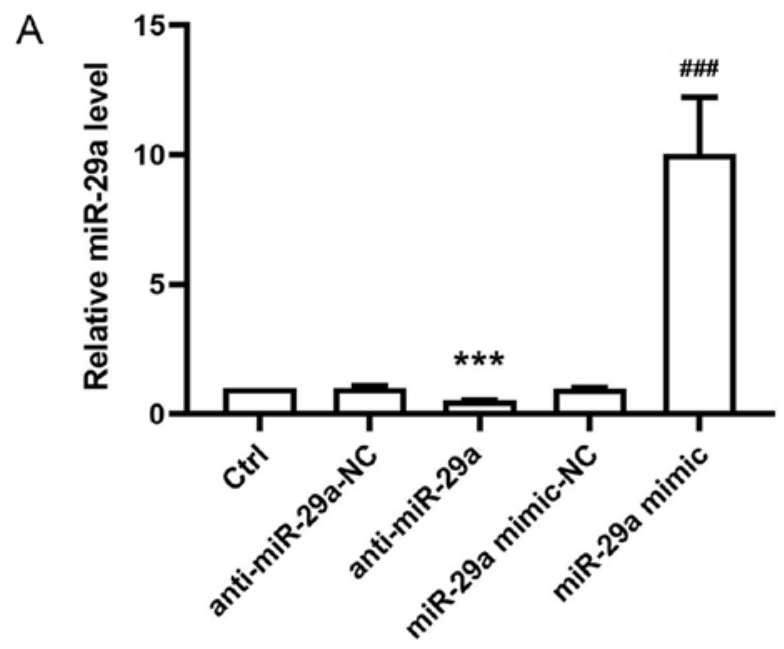

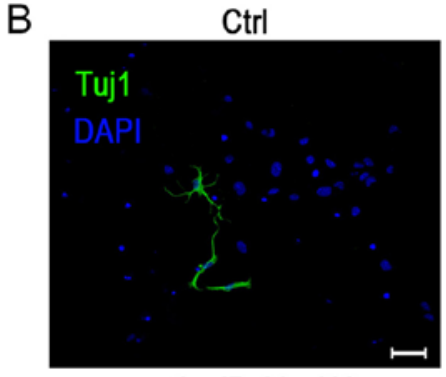

anti-miR-29a-NC

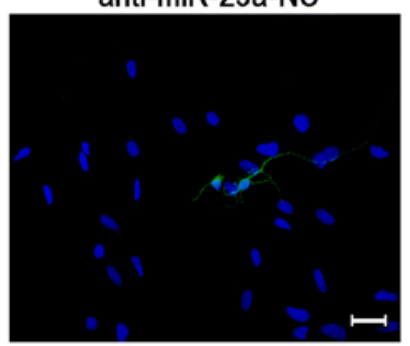

D

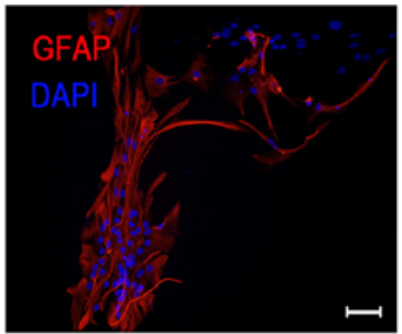

anti-miR-29a-NC

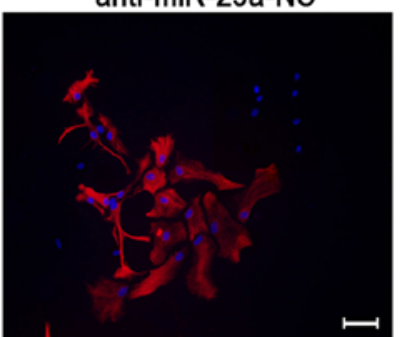

miR-29a mimic-NC

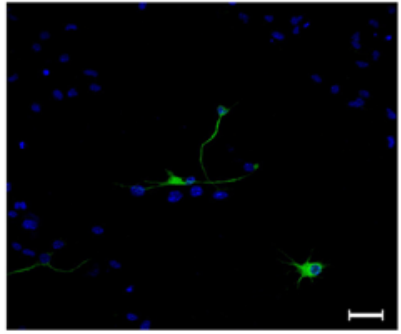

anti-miR-29a

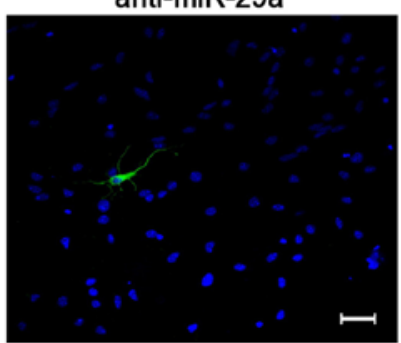

miR-29a mimic-NC

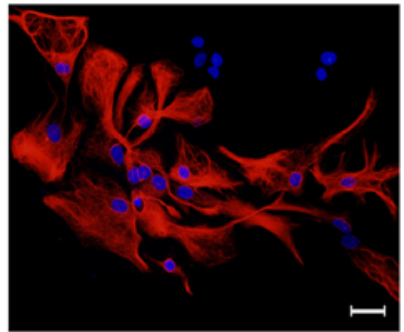

anti-miR-29a

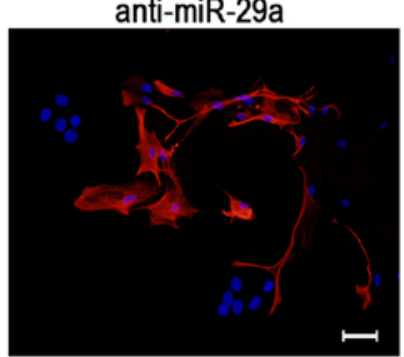

miR-29a mimic
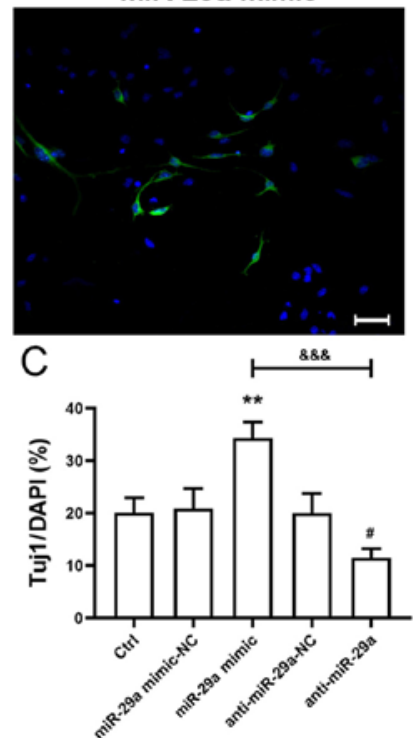

miR-29a mimic
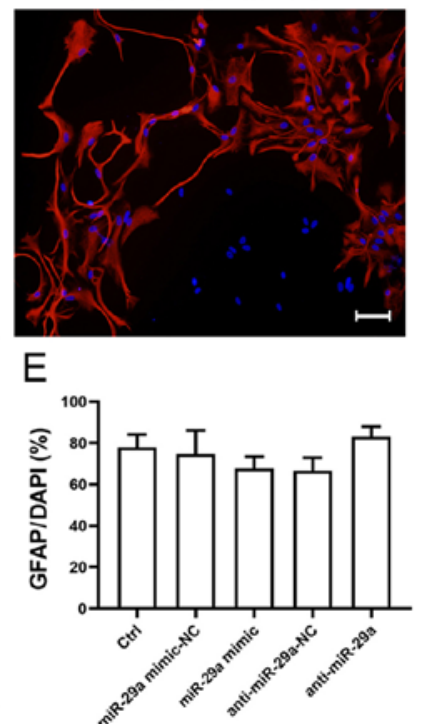

Figure 2. Overexpression of miR-29a increases the number of Tuj1-positive cells. Single neural stem/progenitor cells were transfected with miR-29a mimic-NC, miR-29a mimic, anti-miR-29a or anti-miR-29a-NC and cultured in the differentiation medium for 3 days. (A) Expression levels of miR-29a in each group were analyzed using reverse transcription-quantitative PCR analysis. ${ }^{* * *} \mathrm{P}<0.001$ vs. anti-miR-29a-NC. ${ }^{\# \# "} \mathrm{P}<0.001$ vs. miR-29a mimic-NC. (B) Tuj1-positive cells were identified in each group using immunofluorescent staining. (C) Quantification of part (B). (D) GFAP-positive cells were detected in each group using immunofluorescent staining. (E) Quantification of part (D). Scale bar, $50 \mu \mathrm{m}$. Data from three independent experiments are presented as the percentage of Tuj1- or GFAP-positive cells in the total population of DAPI cells. ${ }^{* *} \mathrm{P}<0.01$ vs. miR-29a mimic-NC. ${ }^{~} \mathrm{P}<0.05$ vs. anti-miR-29a-NC. ${ }^{\text {\&\&\& }} \mathrm{P}<0.001$ vs. miR-29a mimic. miR, microRNA; Tuj1, neuron-specific class III $\beta$-tubulin; GFAP, glial fibrillary acidic protein; NC, negative control; Ctrl, control. 
A

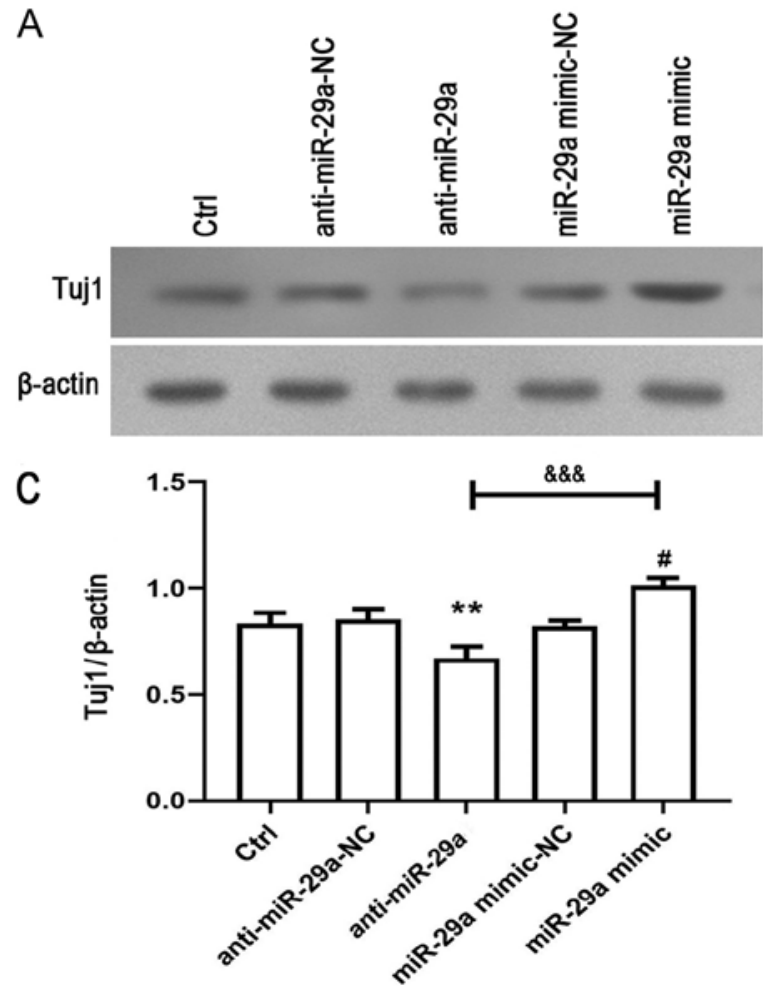

B

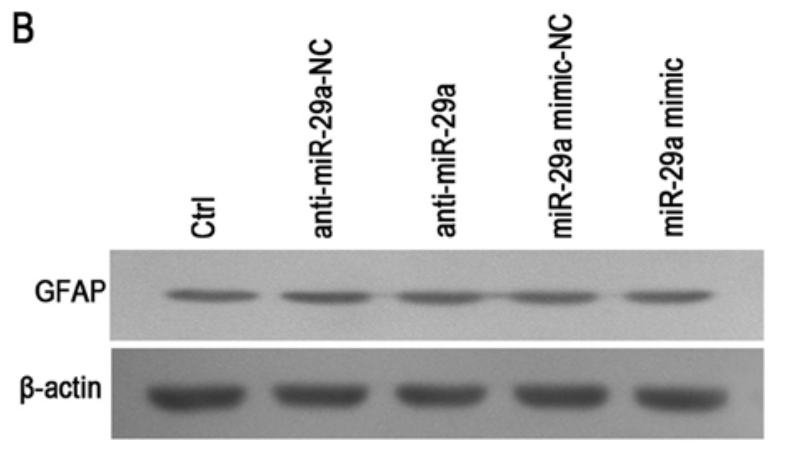

D

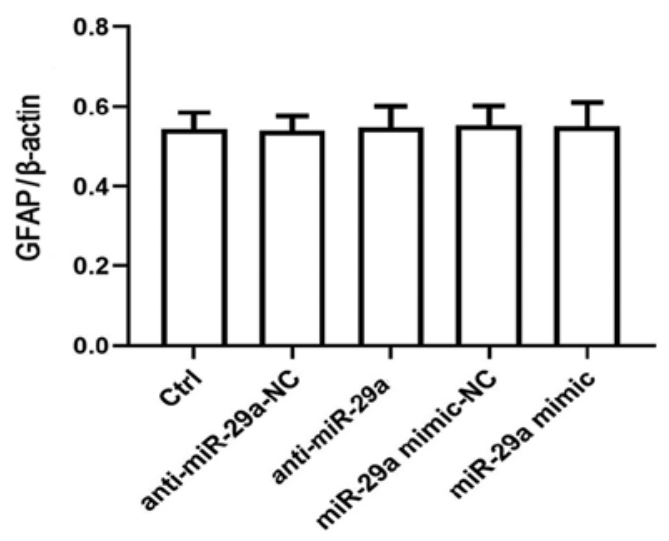

Figure 3. Overexpression of miR-29a upregulates the expression levels of Tuj1. Single neural stem/progenitor cells were transfected with miR-29a mimic-NC, miR-29a mimic, anti-miR-29a or anti-miR-29a-NC and cultured in the differentiation medium for 3 days. (A) Western blotting was used to analyze the expression levels of Tuj1 and (B) GFAP in each group. (C) Semi-quantification of the protein expression levels presented in part (A). (D) Semi-quantification of the protein expression levels presented in part (B). The data are presented as the mean \pm SD of three independent experiments. ${ }^{* * *}<0.01$ vs. anti-miR-29a-NC. ${ }^{\#} \mathrm{P}<0.05$ vs. miR-29a mimic-NC. \&\&\& $\mathrm{P}<0.001$ vs. anti-miR-29a. miR, microRNA; Tuj1, neuron-specific class III $\beta$-tubulin; GFAP, glial fibrillary acidic protein; $\mathrm{NC}$, negative control; Ctrl, control.

of miR-29a were significantly increased compared with the miR-NC group, whereas the anti-miR-29a group demonstrated the opposite trend compared with the anti-miR-29a-NC group (Fig. 2A). These findings indicated that both the miR-29a mimic and anti-miR-29a had a significant effect on the miR-29a expression levels in NSPCs. Following the transfection, Tuj1 and GFAP immunofluorescence staining was performed to determine the impact of miR-29a on NSPC differentiation. Tuj1 and GFAP immunofluorescence staining observed that there were no significant differences in the expression of Tuj1 or GFAP between the Ctrl and miR-29a mimic-NC or anti-miR-29a-NC groups (Fig. 2B-E), which suggested that the transfection did not impact the differentiation of NSPCs. Furthermore, compared with the miR-29a mimic-NC, the overexpression of miR-29a significantly increased the number of Tuj1-positive cells (Fig. 2B and C). In contrast, the number of Tuj1-positive cells was decreased by inhibiting the expression of miR-29a compared with the anti-miR-29a NC (Fig. 2B and C). Notably, despite the increased or decreased expression levels of miR-29a achieved by the miR-29a mimic or anti-miR-29a, respectively, the number of GFAP-positive cells was not altered across the groups (Fig. 2D and E).

To further investigate the effects of miR-29a on NSPC differentiation, the expression levels of Tuj1 and GFAP were detected using western blotting. Similar to the immunofluorescence staining results, the overexpression of miR-29a increased the expression levels of Tuj1 compared with the miR-29a mimic-NC, whereas the downregulation of
miR-29a expression levels demonstrated the opposite trend compared with the anti-miR-29a-NC (Fig. 3A and C). Notably, no significant differences were observed in the expression levels of GFAP across all groups following the overexpression or downregulation of miR-29a (Fig. 3B and D). These results indicated that miR-29a may promote neural differentiation, while having no impact on neuroglial differentiation.

miR-29a modulates the expression levels of KLF4 following NSPC differentiation. KLF4, as a member of the KLFs, has been reported to be involved in a number of cellular processes, including proliferation, differentiation and survival $(19,20)$. Thus, the expression levels of KLF4 during NSPC differentiation were determined using RT-qPCR. The results demonstrated that the expression levels of KLF4 were downregulated in a time-dependent manner (Fig. 4A). To determine whether KLF4 was a direct target gene of miR-29a, the possible targets of miR-18a were predicted using TargetScan 6.2 (http://www. targetscan.org/vert_61/) and miRDB databases (http://mirdb. org/). The results showed that the KLF4 3'UTR containing the predicted miR-29a-binding site was co-transfected into NSPCs to analyze the relative luciferase activity in the presence of the miR-29a mimic, anti-miR-29a, and negative control miRNA. The predicted binding site for miR-29a in the KLF4 3'UTR is presented in Fig. 4B. As KLF4 was found to be targeted by miR-200c (21), exogenous miR-200c was used as a control to repress the KLF4 3'UTR and a dual-luciferase reporter assay and western blotting were performed. Dual-luciferase 


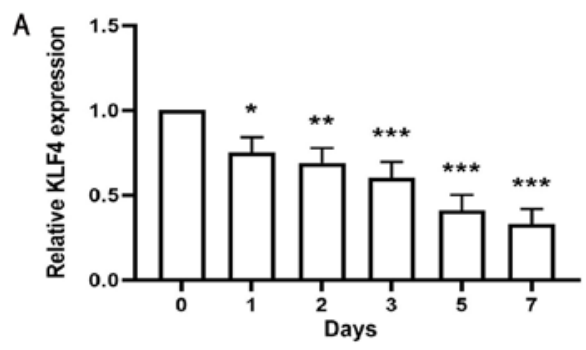

B

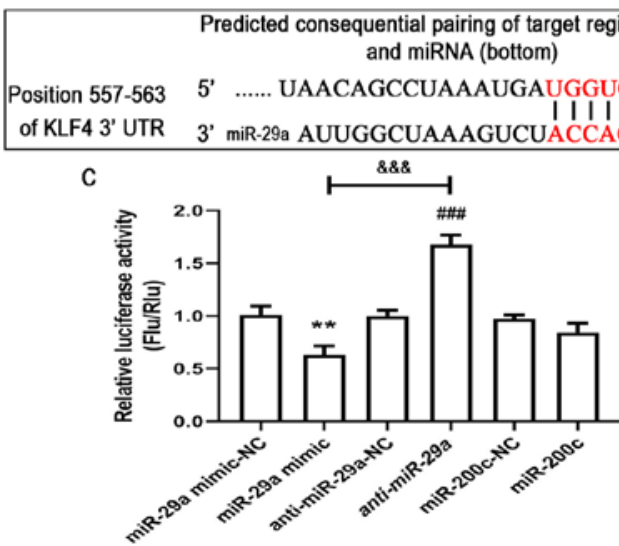

$\mathrm{F}$
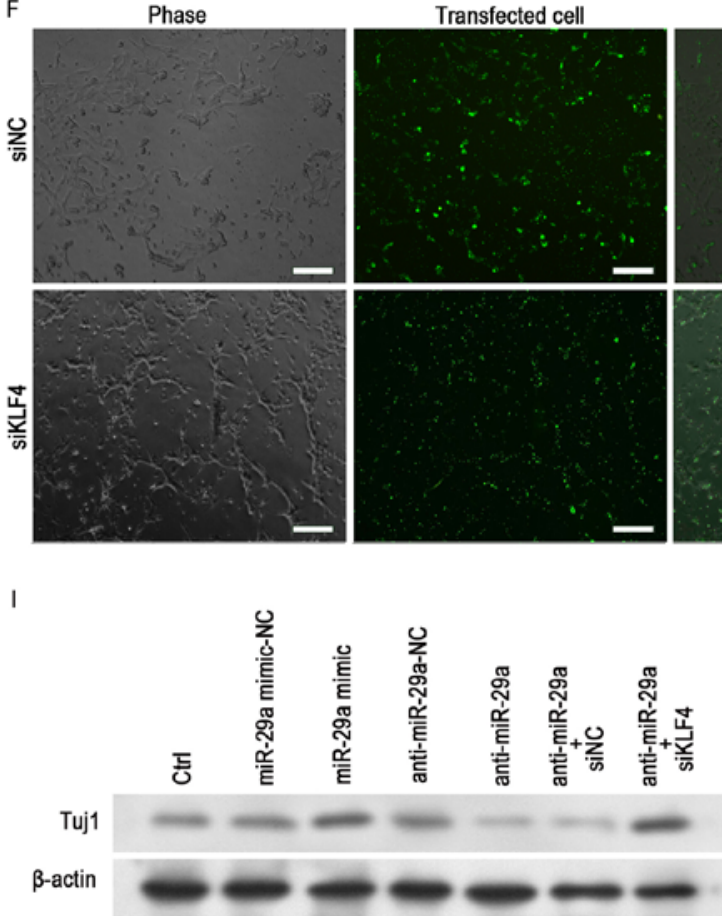

D

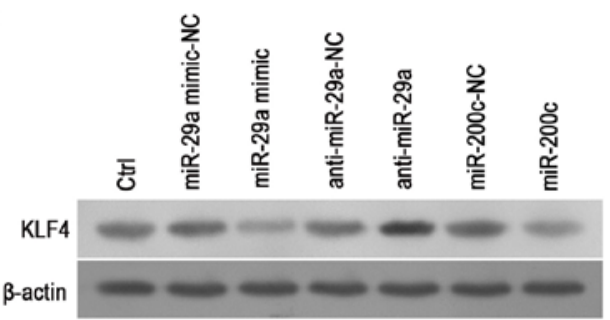

E

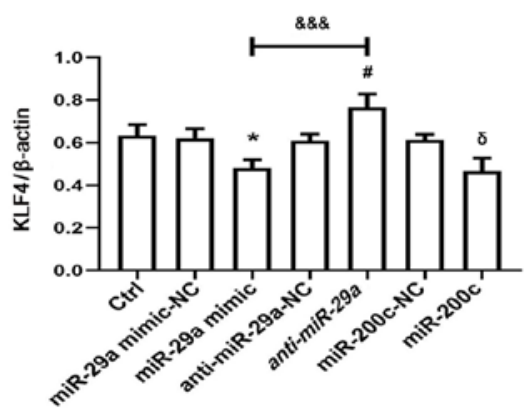

Merged
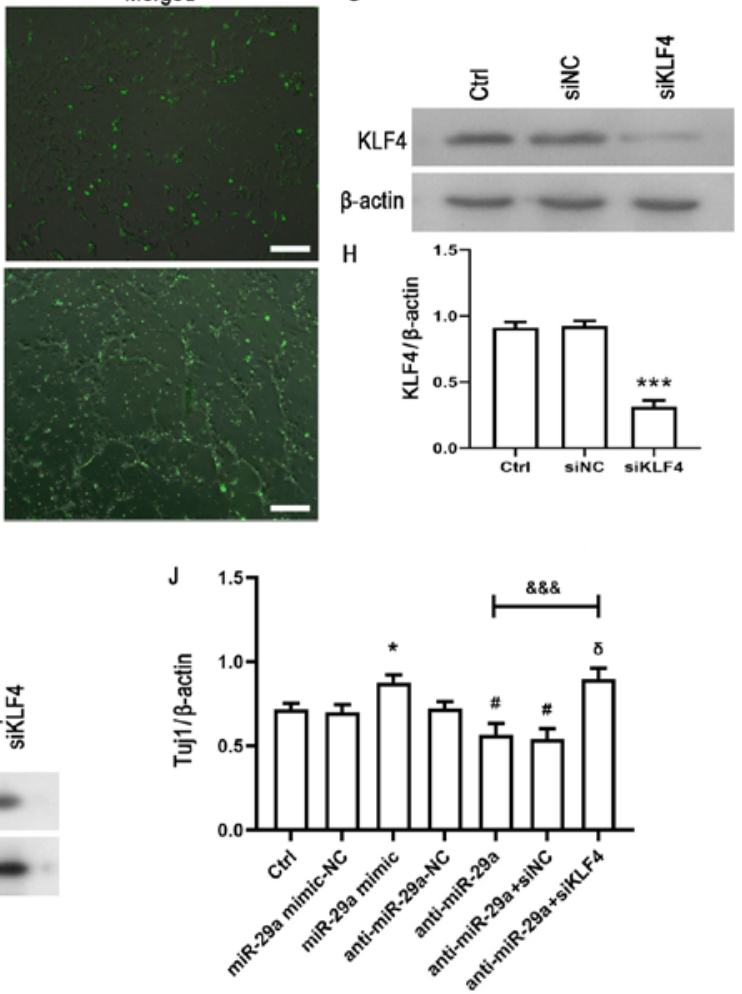

Figure 4. miR-29a regulates the expression levels of KLF4. (A) NSPCs were cultured in normal differentiation medium and the expression levels of KLF4 were analyzed using reverse transcription-quantitative PCR at different time points. ${ }^{*} \mathrm{P}<0.05,{ }^{* *} \mathrm{P}<0.01,{ }^{* * *} \mathrm{P}<0.001$ vs. control $(0 \mathrm{~d})$. (B) Potential binding region of miR-29a on the KLF4 3'UTR was predicted using TargetScan. (C) Dual-luciferase activity was performed to determine the relative luciferase activity of NSPCs co-transfected with the KLF4 3'UTR and the miR-29a mimic-NC, miR-29a mimic, anti-miR-29a, anti-miR-29a-NC, miR-200c-NC or miR-200c. ${ }^{* *} \mathrm{P}<0.01$ vs. miR-29a mimic-NC. ${ }^{\# \# P}$ P 0.05 vs. anti-miR-29a-NC. ${ }^{\& \& \&} \mathrm{P}<0.001$ vs. miR-29a mimic. (D) Single NSPCs were transfected with miR-29a mimic-NC, miR-29a mimic, anti-miR-29a, anti-miR-29a-NC, miR-200c (positive control) or miR-200c-NC and cultured in the differentiation medium for 3 days. Western blotting was used to analyze the expression levels of KLF4. (E) Semi-quantification of the protein expression levels presented in part (D). The data are presented as the mean \pm SD of three independent experiments. ${ }^{*} \mathrm{P}<0.05$ vs. miR-29a mimic-NC. ${ }^{\sharp} \mathrm{P}<0.05$ vs. anti-miR-29a-NC. ${ }^{\circ} \mathrm{P}<0.05$ vs. miR-200c-NC. ${ }^{\& \&} \mathrm{P}<0.001$ vs. miR-29a mimic. (F) NSPCs were transfected for $6 \mathrm{~h}$ with FAM-labeled non-specific siNC or siKLF4 using Lipofectamine ${ }^{\circledR} 2000$. An equal volume of medium was added to the control group. On the second day, $>90 \%$ of cells were observed to be transfected (green). Scale bar, $100 \mu \mathrm{m}(\mathrm{G}) \mathrm{Western}$ blotting analysis and (H) semi-quantification demonstrated that siKLF4 reduced the expression levels of KLF4. ${ }^{* * *} \mathrm{P}<0.001 \mathrm{vs}$. the siNC group. (I) Following the transfection of NSPCs with miR-29a mimic-NC, miR-29a mimic, anti-miR-29a, anti-miR-29a-NC or anti-miR-29a + siKLF4, NSPCs were cultured in the differentiation medium for 3 days and Tuj1 expression levels were detected using western blotting. (J) Semi-quantification of the protein expression levels presented in part (I). The data are presented as the mean $\pm \mathrm{SD}$ of three independent experiments. ${ }^{*} \mathrm{P}<0.05$ vs. miR-29a mimic-NC. ${ }^{\#} \mathrm{P}<0.05$ vs. anti-miR-29a-NC. ${ }^{\circ} \mathrm{P}<0.05$ vs. anti-miR-29a-NC+siNC. ${ }^{2} \& \mathrm{P}<0.001$ vs. anti-miR-29a. KLF4, Kruppel-like factor 4; miR/miRNA, microRNA; UTR, untranslated region; NC, negative control; NSPCs, neural stem/progenitor cells; Flu/Rlu, Firefly luciferase activity/Renilla luciferase activity; Ctrl, control; Tuj1, neuron-specific class III $\beta$-tubulin; si, small interfering RNA. 
reporter assay demonstrated that miR-29a mimic significantly decreased luciferase activity, whereas anti-miR-29a had the opposite effect (Fig. 4C). Furthermore, overexpression of miR-29a significantly downregulated the expression levels of KLF4 compared with the miR-29a mimic-NC group (Fig. 4D and E). Conversely, significantly upregulated expression levels of KLF4 were observed in the anti-miR-29a group compared with the anti-miR-29a-NC group (Fig. 4D and E). To determine whether KLF4 regulated the effects of miR-29a on differentiation, the expression level of KLF4 was knocked down using target siRNA. The siRNA were effectively transfected into NSPC cultures and significantly reduced the expression levels of KLF4 (Fig. 4F-H). Subsequently, KLF4 expression levels were knocked down in the anti-miR-29a group; the inhibition of KLF4 expression levels reversed the effects of anti-miR-29a on the expression levels of Tuj1 (Fig. 4I and J). These data indicated that miR-29a may modulate the expression levels of KLF4.

\section{Discussion}

Previous research indicated that miR-29a had been discovered to serve an important role in numerous biological processes through regulating its target genes; it has been reported to impact proliferation, apoptosis, invasion and oncogenesis $(10,22)$. Increasing evidence has suggested that miR-29a may also be involved in regulating different types of stem cells, including embryonic (11), mesenchymal (23) and hematopoietic stem cells (24). The present study indicated that the overexpression of miR-29a may promote neural differentiation, as the expression levels of Tuj1 were found to be increased. The induction and differentiation of NSPCs into functional neurons have been identified as important steps in the treatment of CNS disease (25). Therefore, the results of the present study suggested that miR-29a may be a novel therapeutic target to treat disorders of the CNS.

The current study demonstrated that miR-29a promoted neural differentiation in cultured rat NSPCs; however, miR-29a was not found to have a role in neuroglial differentiation. It was hypothesized that the excessive percentage of GFAP-positive cells may offer a possible alternative explanation for these findings; the percentage of GFAP-positive cells remained relatively high following the transfection of the miR-29a mimic, leading to little impact on the statistical results.

Accumulating evidence has reported that the miR-29 family target the expression of particular proteins that are significantly involved in disease pathogenesis; for example, miR-29a was reported to be a candidate biomarker for Alzheimer's disease (AD) and Parkinson's disease (PD), due to the abnormal expression levels of miR-29a identified in both AD and PD (26,27). Furthermore, the miR-29b cluster was observed to be decreased in patients with AD, where it participated in regulating the expression levels of $\beta$-secretase 1 , which subsequently promoted the overproduction of amyloid $\beta$ (28). miR-29c was also reported to be involved in the regulation of cerebral ischemia-induced cell death, which depended on its ability to influence the expression levels of RE1-silencing transcription factor and DNA (cytosine-5)-methyltransferase 3A (29). The present study also indicated that miR-29a may promote neuronal differentiation in cultured rat NSPCs, which provided novel insights into the role of miR-29a in neurological disorders and its potential as a therapeutic strategy.

As a zinc finger-containing transcription factor, KLF4 has been found to be expressed in numerous types of mammalian cells, where it regulates diverse cellular processes, such as proliferation, differentiation and maintaining stemness (30-32). For example, in a previous study, following the transfection of the four genes, Oct4, Sox2, KLF4 and c-Myc, somatic cells were reprogrammed and differentiated into induced pluripotent stem cells $(33,34)$. The current study demonstrated that miR-29a downregulated the expression levels of KLF4, which were detected using RT-qPCR and western blotting. These findings suggested that miR-29a may influence neural differentiation by regulating KLF4, which provided a novel insight into the potential function of KLF4 in NSPCs. However, it should be noted that the overexpression of miR-29a may reside in the potential lack of faultless control, which manifests as an increased risk of cancer or differentiation into unpredicted cell phenotype. Therefore, additional research is required to determine the precise mechanism by which miR-29a regulates the neural differentiation of NSPCs.

The present study demonstrated that miR-29a promotes neuronal differentiation in cultured rat NSPCs via regulation of KLF4 factor. The present study also provides a novel insight into potential treatment strategies for CNS damage.

\section{Acknowledgements}

Not applicable.

\section{Funding}

The present study was supported by the National Natural Science Foundation of China (grant no. 81000030) and the Fundamental Research Funds for the Central Universities (grant no. xzy012019104).

\section{Availability of data and materials}

The datasets used and/or analyzed during the current study are available from the corresponding author on reasonable request.

\section{Authors' contributions}

YG, HQ, TZ and YH designed the experiments; YH supervised the research; YG, HQ and ZL performed the majority of the experiments; YG drafted the manuscript; and HQ and YH revised the manuscript. All authors read and approved the final manuscript.

\section{Ethics approval and consent to participate}

All experimental protocols were approved by the Animal Care and Use Regulation of Xi'an Jiaotong University Health Science Center.

\section{Patient consent for publication}

Not applicable. 


\section{Competing interests}

The authors declare that they have no competing interests.

\section{References}

1. Ming GL and Song H: Adult neurogenesis in the mammalian brain: Significant answers and significant questions. Neuron 70: 687-702, 2011

2. Zhao C, Deng W and Gage FH: Mechanisms and functional implications of adult neurogenesis. Cell 132: 645-660, 2008.

3. Sierra A, Encinas JM and Maletic-Savatic M: Adult human neurogenesis: From microscopy to magnetic resonance imaging. Front Neurosci 5: 47, 2011.

4. Abati E, Bresolin N, Comi GP and Corti S: Preconditioning and cellular engineering to increase the survival of transplanted neural stem cells for motor neuron disease therapy. Mol Neurobiol 56: 3356-3367, 2019.

5. Bellenchi GC, Volpicelli F, Piscopo V, Perrone-Capano C and di Porzio U: Adult neural stem cells: An endogenous tool to repair brain injury? J Neurochem 124: 159-167, 2013.

6. Encinas JM and Fitzsimons CP: Gene regulation in adult neura stem cells. Current challenges and possible applications. Adv Drug Deliv Rev 120: 118-132, 2017.

7. Imayoshi I and Kageyama R: The role of Notch signaling in adult neurogenesis. Mol Neurobiol 44: 7-12, 2011.

8. Ming GL and Song H: Adult neurogenesis in the mammalian central nervous system. Annu Rev Neurosci 28: 223-250, 2005.

9. Meza-Sosa KF, Pedraza-Alva G and Pérez-Martínez L: microRNAs: Key triggers of neuronal cell fate. Front Cell Neurosci 8: 175, 2014

10. Alizadeh M, Safarzadeh A, Beyranvand F, Ahmadpour F, Hajiasgharzadeh K, Baghbanzadeh A and Baradaran B: The potential role of miR-29 in health and cancer diagnosis, prognosis, and therapy. J Cell Physiol 234: 19280-19297, 2019.

11. Rajendran G, Dutta D, Hong J, Paul A, Saha B, Mahato B, Ray S, Home P, Ganguly A, Weiss ML and Paul S: Inhibition of protein kinase $\mathrm{C}$ signaling maintains rat embryonic stem cell pluripotency. J Biol Chem 288: 24351-24362, 2013.

12. Gao Y, Qiao H, Lu Z and Hou Y: miR29 promotes the proliferation of cultured rat neural stem/progenitor cells via the PTEN/AKT signaling pathway. Mol Med Rep 20: 2111-2118, 2019.

13. Zhang Z, Zheng X, Liu Y, Luan Y, Wang L, Zhao L, Zhang J, Tian Y, Lu H, Chen X and Liu Y: Activation of metabotropic glutamate receptor 4 regulates proliferation and neural differentiation in neural stem/progenitor cells of the rat subventricular zone and increases phosphatase and tensin homolog protein expression. J Neurochem: Feb 12, 2020 doi: 10.1111/jnc.14984. Online ahead of print

14. Chen X, Tian Y, Yao L, Zhang J and Liu Y: Hypoxia stimulates proliferation of rat neural stem cells with influence on the expression of cyclin D1 and c-Jun N-terminal protein kinase signaling pathway in vitro. Neuroscience 165: 705-714, 2010

15. Jia L, Chopp M, Wang L, Lu X, Zhang Y, Szalad A and Zhang ZG: MiR-34a regulates axonal growth of dorsal root ganglia neurons by targeting FOXP2 and VAT1 in postnatal and adult mouse. Mol Neurobiol 55: 9089-9099, 2018.

16. Livak KJ and Schmittgen TD: Analysis of relative gene expression data using real-time quantitative PCR and the 2(-Delta Delta C(T)) method. Methods 25: 402-408, 2001.

17. Zhang Z, Hu F, Liu Y, Ma B, Chen X, Zhu K, Shi Y, Wei T, Xing Y, Gao Y, et al: Activation of type 5 metabotropic glutamate receptor promotes the proliferation of rat retinal progenitor cell via activation of the PI-3-K and MAPK signaling pathways. Neuroscience 322: 138-151, 2016.
18. Suzuki S, Namiki J, Shibata S, Mastuzaki $\mathrm{Y}$ and Okano $\mathrm{H}$ : The neural stem/progenitor cell marker nestin is expressed in proliferative endothelial cells, but not in mature vasculature. J Histochem Cytochem 58: 721-730, 2010.

19. Takahashi $\mathrm{K}$ and Yamanaka S: A decade of transcription factor-mediated reprogramming to pluripotency. Nat Rev Mol Cell Biol 17: 183-193, 2016.

20. Black AR, Black JD and Azizkhan-Clifford J: Sp1 and krüppel-like factor family of transcription factors in cell growth regulation and cancer. J Cell Physiol 188: 143-160, 2001.

21. Wellner U, Schubert J, Burk UC, Schmalhofer O, Zhu F, Sonntag A, Waldvogel B, Vannier C, Darling D, zur Hausen A, et al: The EMT-activator ZEB1 promotes tumorigenicity by repressing stemness-inhibiting microRNAs. Nat Cell Biol 11: 1487-1495, 2009.

22. van Rooij E and Olson EN: MicroRNA therapeutics for cardiovascular disease: Opportunities and obstacles. Nat Rev Drug Discov 11: 860-872, 2012.

23. Jin $\mathrm{M}$, Wu Y, Wang $\mathrm{J}$, Ye W, Wang L, Yin $\mathrm{P}$, Liu W, Pan C and Hua X: MicroRNA-29 facilitates transplantation of bone marrow-derived mesenchymal stem cells to alleviate pelvic floor dysfunction by repressing elastin. Stem Cell Res Ther 7: 167, 2016.

24. O'Connell RM: Endogenous miR-29a regulates HSC function in mammals. Blood 125: 2180-2181, 2015.

25. Barkho BZ and Zhao X: Adult neural stem cells: Response to stroke injury and potential for therapeutic applications. Curr Stem Cell Res Ther 6: 327-338, 2011.

26. Müller M, Jäkel L, Bruinsma IB, Claassen JA, Kuiperij HB and Verbeek MM: MicroRNA-29a is a candidate biomarker for Alzheimer's disease in cell-free cerebrospinal fluid. Mol Neurobiol 53: 2894-2899, 2016

27. Bai X, Tang Y, Yu M, Wu L, Liu F, Ni J, Wang Z, Wang J, Fei J, Wang W, et al: Downregulation of blood serum microRNA 29 family in patients with Parkinson's disease. Sci Rep 7: 5411, 2017.

28. Hébert SS, Horré K, Nicolaï L, Papadopoulou AS, Mandemakers W, Silahtaroglu AN, Kauppinen S, Delacourte A and De Strooper B: Loss of microRNA cluster miR-29a/b-1 in sporadic Alzheimer's disease correlates with increased BACE1/beta-secretase expression. Proc Natl Acad Sci USA 105: 6415-6420, 2008

29. Pandi G, Nakka VP, Dharap A, Roopra A and Vemuganti R: MicroRNA miR-29c down-regulation leading to de-repression of its target DNA methyltransferase 3a promotes ischemic brain damage. PLoS One 8: e58039, 2013.

30. Rowland BD and Peeper DS: KLF4, p21 and context-dependent opposing forces in cancer. Nat Rev Cancer 6: 11-23, 2006.

31. Ghaleb AM and Yang VW: Krüppel-like factor 4 (KLF4): What we currently know. Gene 611: 27-37, 2017.

32. Simmen RCM, Pabona JMP, Velarde MC, Simmons C, Rahal O and Simmen FA: The emerging role of Krüppel-like factors in endocrine-responsive cancers of female reproductive tissues. J Endocrinol 204: 223-231, 2010.

33. Tamanini S, Comi GP and Corti S: In vivo transient and partial cell reprogramming to pluripotency as a therapeutic tool for neurodegenerative diseases. Mol Neurobiol 55: 6850-6862, 2018.

34. Vangapandu H and Ai W: Krüppel like factor 4 (KLF4): A transcription factor with diverse context-dependent functions. Gene Ther Mol Biol 13a: 194-204, 2009.

(i) $\odot$ This work is licensed under a Creative Commons Attribution-NonCommercial-NoDerivatives 4.0 International (CC BY-NC-ND 4.0) License. 